\title{
PARTICLE-IN-CELL SIMULATION CONCERNING HEAT-FLUX MITIGATION USING ELECTROMAGNETIC FIELDS
}

\author{
Lüskow K. F. ${ }^{a, *}$, Kemnitz S. ${ }^{a, c}$, Bandelow G. ${ }^{a}$, Duras J. ${ }^{a}$, \\ KAhnfeld D. ${ }^{a}$, Schneider R. ${ }^{a}$, Konigorski D. ${ }^{b}$ \\ ${ }^{a}$ Ernst-Moritz-Arndt University Greifswald, Institute of Physics, Felix-Hausdorff-Str. 6, 17487 Greifswald, \\ Germany \\ ${ }^{b}$ Airbus Operations GmbH, Emerging Technologies $\&$ Concepts, Kreetslag 10, 21129 Hamburg, Germany \\ ${ }^{c}$ University Rostock, Institute of Informatics, Albert-Einstein-Str. 22, 18059 Rostock, Germany \\ * lueskow@physik.uni-greifswald.de
}

\begin{abstract}
The Particle-in-Cell (PIC) method was used to study heat flux mitigation experiments with argon. In the experiment it was shown that a magnetic field allows to reduce the heat flux towards a target. PIC is well-suited for plasma simulation, giving the chance to get a better basic understanding of the underlying physics. The simulation demonstrates the importance of a self-consistent neutral-plasma description to understand the effect of heat flux reduction.
\end{abstract}

Keywords: Particle-in-Cell, argon, heat-flux mitigation, electromagnetic fields.

\section{Motivation}

In space missions an enormous amount of money is spent for the thermal protection systems for re-entry. To minimize these costs one is interested in finding new ways of reducing the heat flux. The idea is to use magnetic fields to guide the flux away from the re-entry vehicle. In 2002 the European Space Agency (ESA) started an investigation on heat flux mitigation by means of MHD in partially-ionized argon-flows. Argon was chosen for first-principle tests, because it is a noble gas and inert. Therefore, additional complications from complex chemistry - like in air do not appear, simplifying the analysis of such tests. A strong heat flux reduction was measured, created by an externally applied magnetic field [1]. In the past Navier-Stokes fluid codes for the neutrals were applied combined with rather crude models for the plasma components. Such models were not able to reproduce the experimental observations. With the help of a kinetic particle method one expects to get a more realistic description of the system, including neutrals and plasma. Therefore, a Particle-in-Cell code with Monte-Carlo Collisions (PIC-MCC) was developed.

\section{Code description and simulation set-up}

The code uses the well-established Particle-in-Cell method combined with Monte-Carlo collisions (PICMCC). PIC was successfully applied in various fields of plasma physics to obtain a better physical understanding. PIC-MCC simulation delivers a fully self-consistent microscopic description of a plasma. Therefore, all plasma-parameters (macroscopic and microscopic) can be diagnosed. In particular, entities like potential, densities, temperatures, velocity distribution functions and power fluxes can be calculated. A more detailed description of the method itself can be found in different reviews [2, 3].

So-called "super-particles" are used, each of them representing a number of real physical particles. All of them follow the same trajectory due to the fact that they have the same mass-to-charge ratio and therefore the same acceleration by the Lorentz force. The particles move in the self-consistent electric field which is calculated on a spatially equidistant grid from the Poisson equation, which itself is determined by the space charge deposited by the pseudo-particles and the applied boundary conditions. The real mass ratio between electrons and ions is used. Particle collisions are handled by Monte-Carlo collisions (MCC) routines, that change particle-velocities according to the underlying collision dynamics. All relevant collision types are implemented in the code: electron-neutral elastic, ionization, excitation, coulomb collisions, ion-neutral momentum transfer and charge exchange. Neutrals are treated as plasma particles with zero charge. This, in fact, means that the neutral model is a Direct Simulation Monte-Carlo (DSMC) description.

In the model two spatial (axial and radial) and three velocity components $(2 \mathrm{~d} 3 \mathrm{v})$ are resolved. The computational domain is a region of $42 \mathrm{~cm}$ in axial and $14 \mathrm{~cm}$ in radial direction. It consists of a particle source on the left boundary and a target on the right, both lying directly at the symmetry axis. All particles crossing the symmetry axis get reflected. The face of the blunt body target is placed at $26 \mathrm{~cm}$, it has a radius of $3.6 \mathrm{~cm}$. The blunt body is implemented as metallic, but is covered by a dielectric surface made of quartz $(\epsilon=4.27)$. The left domain boundary has a fixed potential of $0.5 \mathrm{~V}$ to represent the exit of the arc- 


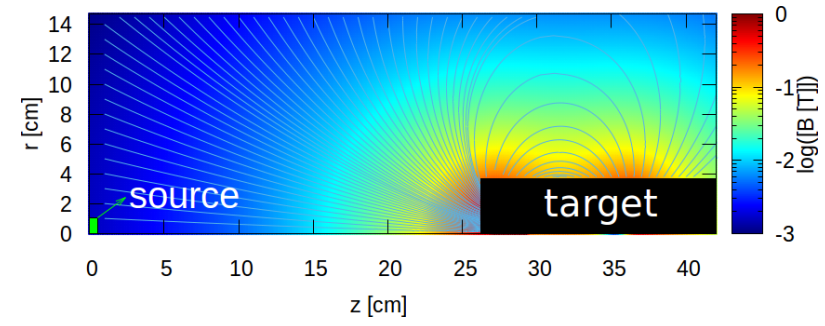

Figure 1. Magnetic field used for the current simulation domain.

jet used for the experiment and ensures that particles injected here move towards the target. The upper and the right domain boundaries are set to zero potential.

The magnetic field was calculated using the FiniteElement Magnetic Method solver (FEMM) 4]. The topology of the dipole-like magnetic field is shown in figure 1

At outer domain boundaries all particles get absorbed. The same procedure is done for plasma particles at the target. Secondary electron emission can be neglected due to the small emission coefficient of quartz $(<0.5)$ at low energies [5]. Neutrals are reflected at the target surface, because it is assumed that noble gases are inert to chemical reactions.

A uniform background density is combined with a local particle source at the left side of the domain. The neutral gas density reproduces the background pressure from the experiment that is not created by the source but is an artifact of the non-perfect vacuum creation in the test-chamber.

The number of particles injected in each timestep were adjusted in order to reach the measured density from the experiment $\left(n_{e}=n_{i} \approx 2.8 \cdot 10^{11} \mathrm{~cm}^{-3}\right.$, $\left.n_{n} \approx 1.6 \cdot 10^{15} \mathrm{~cm}^{-3}\right)$. Neutrals are injected with a maxwellian-distributed velocity with a drift of $2070 \mathrm{~m} / \mathrm{s}$ to represent the free stream velocity measured by microwave interferometry. Ions have similar source velocities as neutrals. Electrons are injected with a two temperature energy distribution. The main peak of the distribution function is at $0.7 \mathrm{~V}$ which is the measured averaged electron temperature from experiment. Due to the strong emission of light during experiment one also needs hot tail electrons that are represented in the simulation by a second distribution at higher energies but with lower particles numbers. This is a typical feature of plasma sources, for example in RF plasmas [6]. The high energy tails exist because the mean free path of such electrons are longer than the source dimension and non-Maxwellian distribution functions can sustain. A plot of the energy distribution functions that is injected in the simulation can be seen in figure 2 .

To optimize numerical performance the size of the domain is scaled down by a factor of 100 preserving the ratios of system lengths to gyroradii and mean free path. As a consequence of similarity scaling densities and the magnetic field are increased by the same

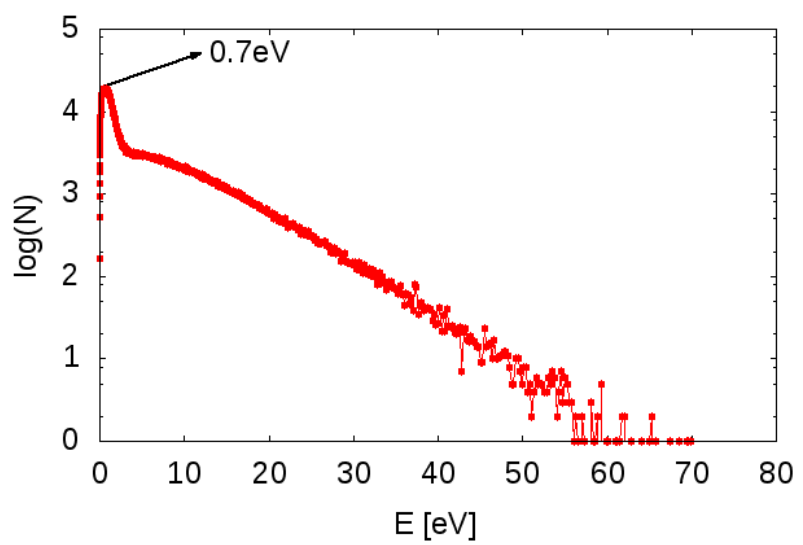

Figure 2. Electron energy distribution injected by the particle source.

factor. This ensures the correct physical behavior of all particles.

The large difference between neutral and plasma density leads to a problem in computational resources due to the large number of neutral particles if the same pseudo-particle factor is applied to neutrals and plasma. Fortunately, the ionization threshold energy is beyond the measured electron temperature, so one can consider the ionization as negligible. Therefore, one is allowed to implement an additional scaling factor for neutral super-particles. For the current simulation an additional weighting factor of $10^{3}$ is applied. The weighting factor linearly increases the cross-section for every collision in which one neutral is involved. The cross section for neutral-neutral collisions is multiplied by the square of the weighting factor.

According to experimental observations there are stronger fluctuations setting in by applying the magnetic field than in the unmagnetized case. To take this into account in the simulation a radial diffusion model was implemented for the magnetized case. A diffusion coefficient of $10 \mathrm{~m}^{2} \mathrm{~s}^{-1}$ is used for plasma particles. For neutral particles a coefficient of $0.1 \mathrm{~m}^{2} \mathrm{~s}^{-1}$ is set.

This estimate is based on diffusion coefficients from magnetic fusion [7. It is well-known that turbulence in a plasma can be created or at least can be enhanced by magnetic fields [8]. Without activating the turbulence all particles move towards the center of the target and the flux will be strongly increased in contrast to the experiment.

\section{Results}

\subsection{Comparison of the simulation with experiment}

To ensure that the simulation represents the correct physics as observed in the experiment, one has to compare the results from the simulation with the measurements from the experiment. Unfortunately, plasma parameters are not well diagnosed in the experimental campaign. One has only line-averaged values for electron temperature and density. The electron den- 

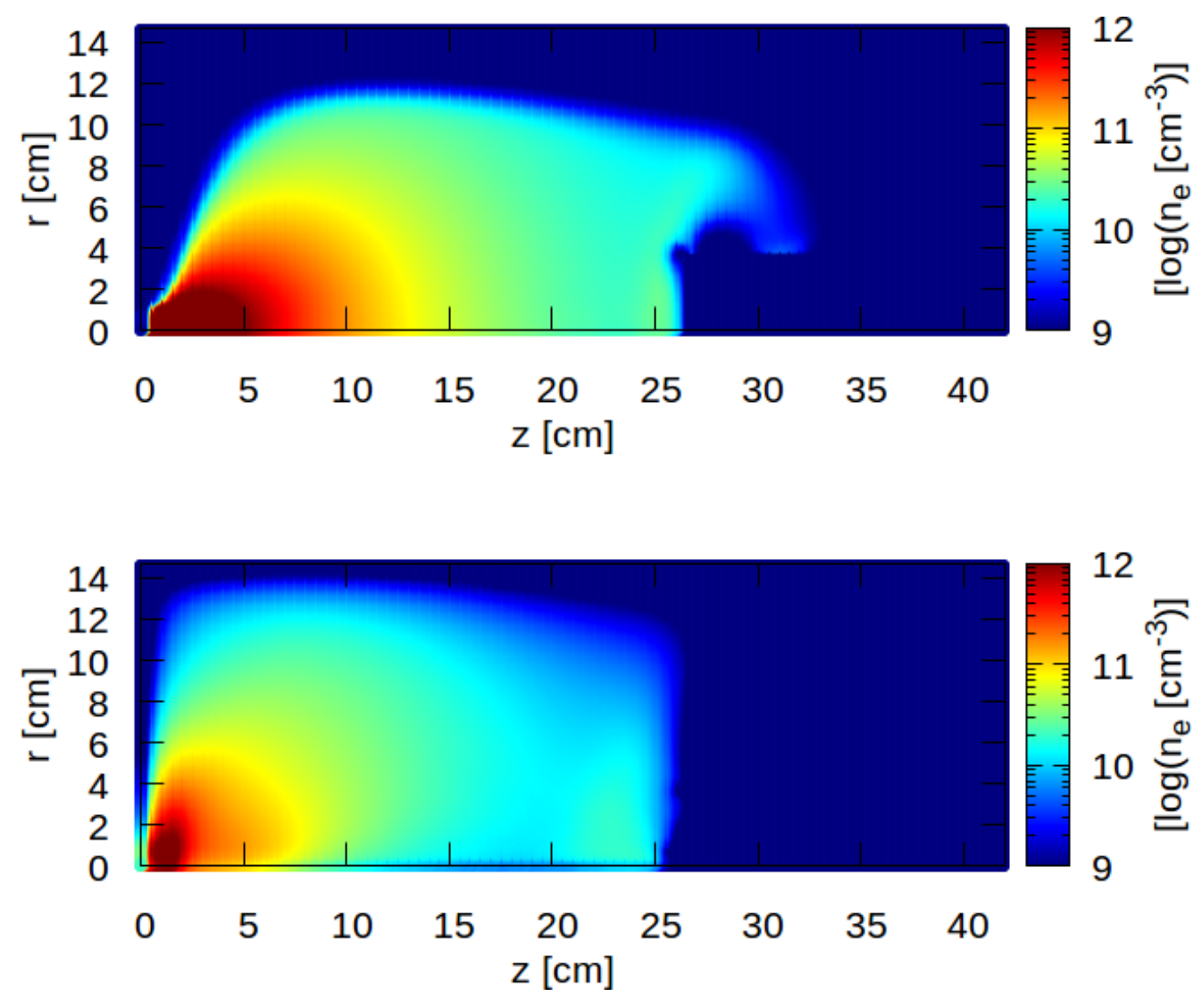

Figure 3. Electron density without (top) and with (bottom) magnetic field.
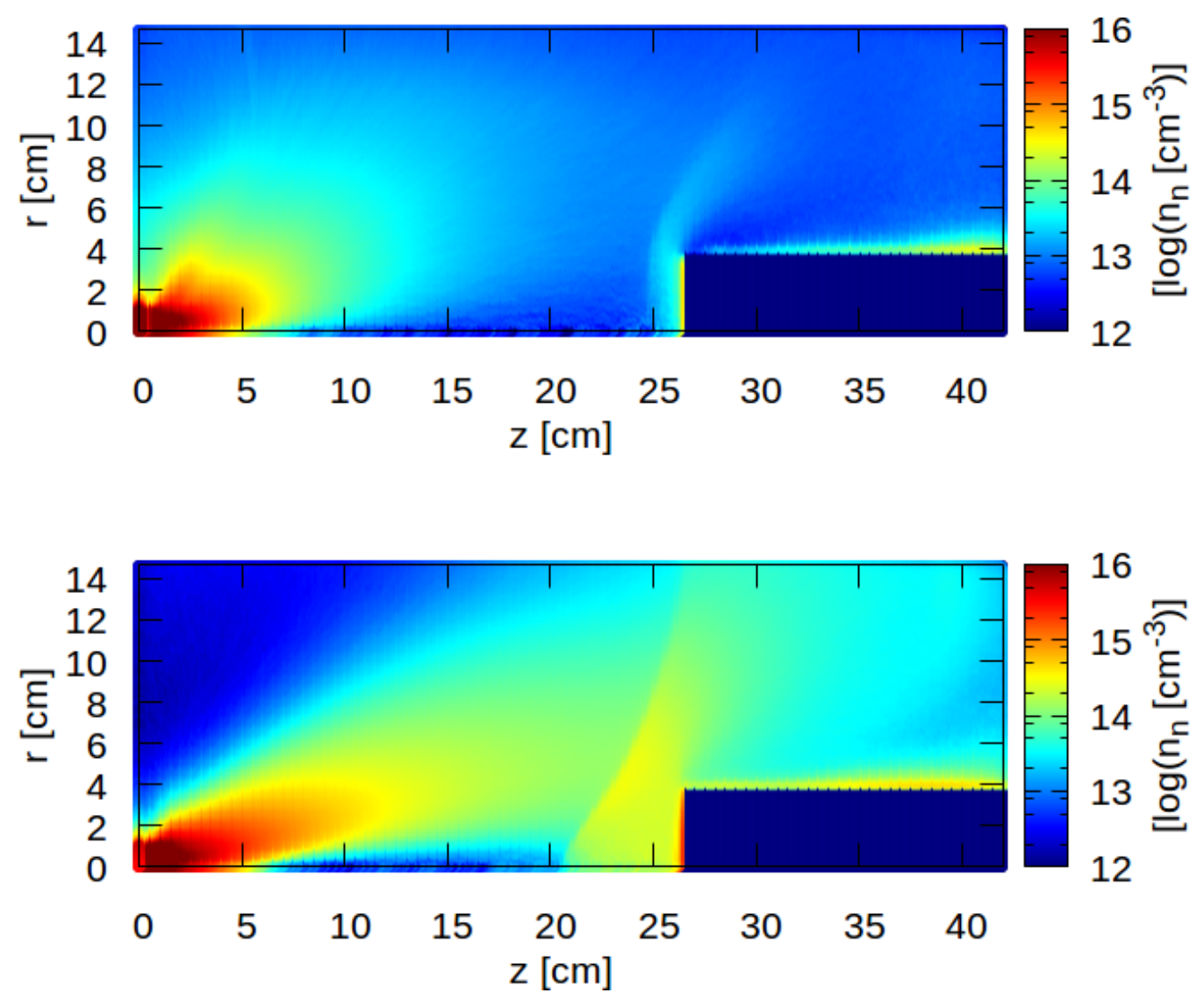

Figure 4. Neutral density without (top) and with (bottom) magnetic field. 

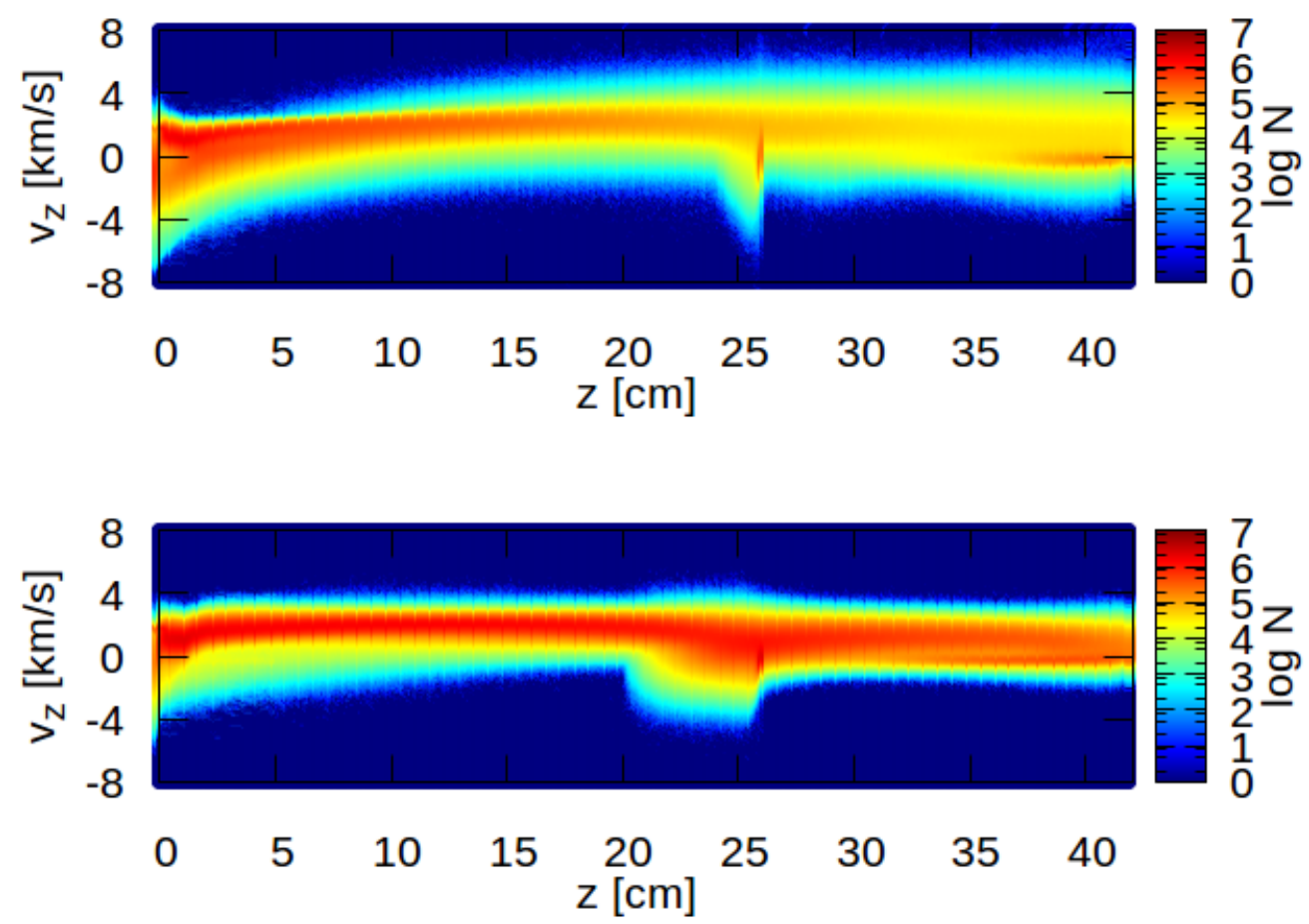

Figure 5. Distribution function of the axial velocity component for neutral particles without (top) and with (bottom) magnetic field.
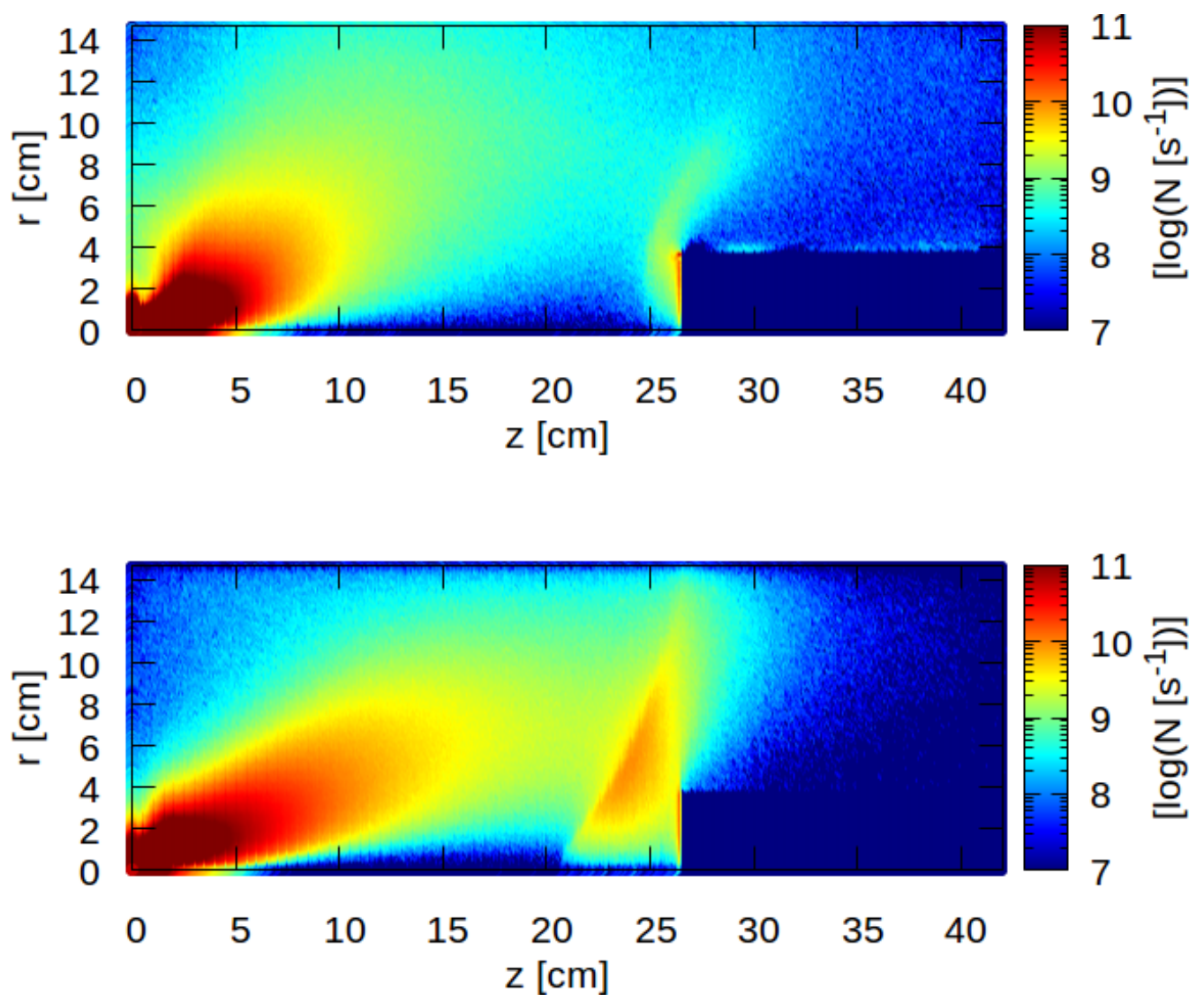

Figure 6. Number of charge exchange collisions per second without (top) and with (bottom) magnetic field. 
sity $n_{e}$ in the experiment was measured by microwave interferometry with the result of $2.8 \cdot 10^{11} \mathrm{~cm}^{-3}[1]$. The simulated density averaged over the region where the measurement takes place is $1.8 \cdot 10^{11} \mathrm{~cm}^{-3}$ for the case without magnetic field. In figure 3 the electron density is shown with and without magnetic field.

In accordance with the experiment one can see a push-back of the region of high electron density from the target. This is in good agreement with the shrinking of the bright emission region from experiment [1]. In addition, the density distribution is changed significantly and in front of the target a region of higher density occurs. Experimentally, an additional bright emission region near the target appears when a magnetic field is applied.

In contrast to plasma parameters the neutral gas dynamics is very well characterized. The averaged neutral density in experiment was $1.6 \cdot 10^{15} \mathrm{~cm}^{-3}$. So it is about 4 orders of magnitude larger than the electron density. The simulated neutral density distribution can be seen in figure 4. In good agreement with the experiment without magnetic field a clear shock front at the target is obtained. The average velocity of neutrals in this region is decreasing from supersonic (about $2500 \mathrm{~m} / \mathrm{s}$ ) to less than $1000 \mathrm{~m} / \mathrm{s}$. The original sharp shock front in the case without magnetic field gets broadened in the case with magnetic field. This broadening is a combination of very strong charge exchange collisions and radial anomalous turbulent transport introduced as described before. The triangular shape of the neutral density in the case with magnetic field is a consequence of the plasma species reacting to the magnetic field. Electrons are magnetized and follow the magnetic field. They get pushed towards the front side of the target, where they accumulate. The increased turbulent transport leads to a radial triangular structure of this increased density region reflecting also the structure of the magnetic fieldlines. Ions follow electrons due to quasi-neutrality and pile up there as well. Charge exchange collisions couple this density increase in the plasma species to the neutrals. Therefore, this triangular structure builds up for neutrals as well. It would be interesting to check, whether this triangular shape in density and the broadening of the shock front is also detectable in experiment. Indications are existing in the experiment, because the shock front practically vanished in the case with magnetic field in the photos (see Figure 6 of [1]).

The simulated axial velocity distribution from DSMC is shown in figure 5 summed over all neutrals with the same axial position independent of their radial position. One can see that the main peak is at about $2000 \mathrm{~m} \mathrm{~s}^{-1}$ similar to the experimentally observed velocity of $2350 \mathrm{~m} \mathrm{~s}^{-1}$. In front of the target there are neutrals with negative axial velocities. These neutrals originate from reflection at the target as determined by collisional effects in the solid (collisional cascades).
The velocity distribution function for ions in Figure 7 looks very similar to the one for neutrals. This resembles the strong coupling by charge exchange collisions.

\subsection{Heat Flux Reduction}

The most important finding from experiment was the strong heat flux mitigation by activating the magnetic field. Due to the relative small electron and ion densities compared to the neutral density one can estimate that most of the heat flux towards the target is carried by the neutrals. Up to now the reason for the strong heat flux reduction by an magnetic field was not understood, because there exists no obvious direct coupling between B-field and neutral particle dynamics.

In the simulation results a significant change in the neutral velocity distribution function appears when the magnetic field is activated. In front of the target there are more particles with negative axial velocities than in the case without magnetic field. An explanation for this can be found in the coupling to the movement of plasma particles.

Electrons moving towards the target experience magnetic field gradients redirecting them towards the axis. As a consequence a redistribution of electron density with an increased density near the axis even in front of the mach cone is appearing. Due to quasineutrality the ion density needs to be similar to the electron density. Argon has the characteristics of very high charge exchange cross-sections. Therefore, the increased ion density in front of the mach cone leads to more charge-exchange collisions that modify the velocity of the neutrals, see figure 6 .

This shows up as an increased region of negative velocities in the axial velocity distributions (see figure 5). As a consequence, the averaged neutral velocity gets reduced compared to the case without magnetic field. The total heat flux profile is summed up over all species contributions (electrons, ions, neutrals). It is dominated in this case by the neutral component. Therefore, less neutral flux reach the target and the total heat flux is reduced, see figure 8 . The chargeexchange collisions act as a parallel momentum sink for the neutral flow slowing it down. The integral reduction of the total heat flux can be calculated by integrating the flux over the target in radial direction. The result is a mitigation to $57 \%$ of the original heat flux without magnetic field. The measured heat flux reduction was $85 \%$ with rather large experimental uncertainties.

\section{Conclusions}

In the frame of this study a PIC-MCC code was developed to simulate a heat flux mitigation experimental campaign performed by DLR 2007. The main result of the experimental campaign was the demonstration to reduce the total heat flux by externally applied 

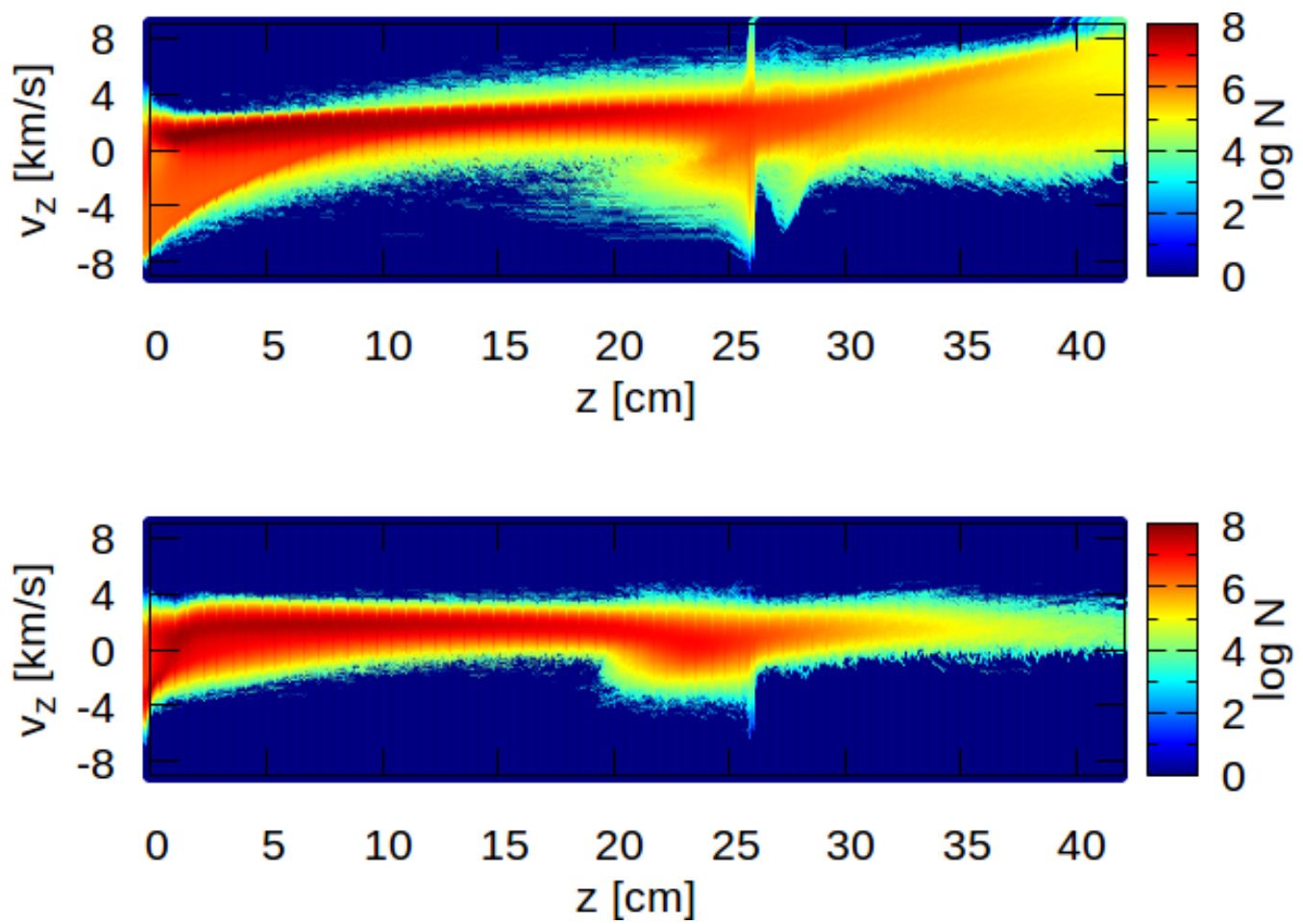

Figure 7. Distribution function of the axial velocity component for ions without (top) and with (bottom) magnetic field.

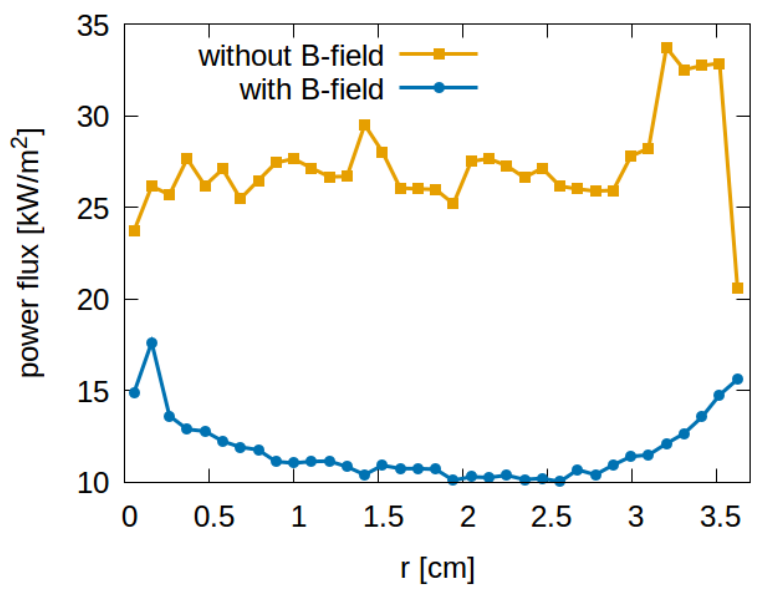

Figure 8. Simulated radial profile of the total heat flux on the target.

magnetic fields. This effect was qualitatively reproduced by PIC-DSMC simulations for the first time. A possible explanation for the effect was found by an increase of charge exchange collisions between plasma ions and neutrals. This shields the target from the impinging neutral particles acting as a momentum sink for the neutral gas flow. The increased ion density driving this effect is also affected by increased radial turbulent transport observed experimentally in the magnetized plasma and taken into account also in the model.

\section{Acknowledgements}

This work was supported by the German Space Agency DLR through Project 50RS1508.

\section{References}

[1] Ali Gülhan, Burkard Esser, Uwe Koch, Frank Siebe, Johannes Riehmer, Domenico Giordano, and Detlev Konigorski. Experimental verification of heat-flux mitigation by electromagnetic fields in partially-ionized-argon flows. Journal of Spacecraft and Rockets, 46(2):274-283, 2009.

[2] Charles K. Birdsall and A. Bruce Langdon. Plasma physics via computer simulation. CRC Press, 2004.

[3] D. Tskhakaya, K. Matyash, R. Schneider, and F. Taccogna. The particle-in-cell method. Contributions to Plasma Physics, 47(8-9):563-594, 2007.

[4] David Meeker. Finite element method magnetics. FEMM, 4:32, 2010.

[5] A Dunaevsky, Y Raitses, and NJ Fisch. Secondary electron emission from dielectric materials of a hall thruster with segmented electrodes. Physics of Plasmas (1994-present), 10(6):2574-2577, 2003.

[6] VA Godyak and RB Piejak. Abnormally low electron energy and heating-mode transition in a low-pressure argon rf discharge at $13.56 \mathrm{mhz}$. Physical review letters, 65(8):996, 1990.

[7] F Perkins, P Barabaschi, D Boucher, JG Cordey, A Costley, J Deboo, PH Diamond, N Fujisawa, CM Greenfield, J Hogan, et al. Iter physics basis. In Plasma Physics and Controlled Nuclear Fusion Research: Proceedings of the... International Conference on Plasma Physics and Controlled Nuclear Fusion Research, volume 15, page 477. International Atomic Energy Agency, 1994.

[8] D. Bohm, E. Burhop, H. S. W. Massey, and R. Williams. The characteristics of electrical discharges in magnetic fields. National nuclear energy series: Electromagnetic Separation Project. McGraw-Hill, 1949. 